\title{
Visit-to-visit variability in systolic blood pressure is a novel risk factor for the progression of coronary artery calcification
}

\author{
Hiroshi Okada ${ }^{1}$, Michiaki Fukui ${ }^{1}$, Muhei Tanaka ${ }^{1}$, Shinobu Matsumoto ${ }^{1}$, Yusuke Mineoka ${ }^{1}$, Naoko Nakanishi ${ }^{1}$, \\ Ki-ichiro Tomiyasu ${ }^{2}$, Koji Nakano ${ }^{3}$, Goji Hasegawa ${ }^{1}$ and Naoto Nakamura ${ }^{1}$
}

Recent studies have suggested that variability in the systolic blood pressure (SBP) is a risk factor for cardiovascular disease (CVD). The aim of this study was to investigate the relationship between variability in the SBP and the progression of coronary artery calcification (CAC), which is a useful marker for CVD. We measured SBP in 164 consecutive patients at every visit over the course of a year and calculated the coefficient of variation and s.d. of the SBP. We performed a follow-up study using multislice computed tomography to assess the progression of the CAC score, the mean interval of which was $3.93 \pm 1.36$ years. We then evaluated the relationship between variability in the SBP and progression of the CAC score. The coefficient of variation for the SBP correlated positively with the progression of the CAC score $(r=0.4382, P<0.0001)$. Multiple regression analysis demonstrated that the coefficient of variation of the SBP $(\beta=0.3826, P<0.0001)$ was independently associated with the progression of the CAC score. The visit-to-visit variability in SBP could be a novel risk factor for the progression of CAC. Hypertension Research (2013) 36, 996-999; doi:10.1038/hr.2013.66; published online 4 July 2013

Keywords: atherosclerosis; coronary artery calcification; variability of blood pressure

\section{INTRODUCTION}

Cardiovascular disease (CVD) is the primary cause of mortality and morbidity in the general population, and several risk factors, including diabetes, smoking, hypertension and dyslipidemia, have been shown to accelerate the progression of CVD. ${ }^{1,2}$ Visit-to-visit variability in the systolic blood pressure (SBP) has been reported to be a strong predictor of CVD. ${ }^{3}$ We reported previously that visit-to-visit variability in the SBP was correlated with atherosclerosis in a crosssectional study. ${ }^{4}$ The coronary artery calcification (CAC) score has recently been developed as a marker of coronary atherosclerosis that can be assessed noninvasively using multislice computed tomography (MSCT). The CAC score is an established quantitative and objective measure of coronary artery atherosclerosis, and several studies have demonstrated a correlation between the CAC score and CVD. ${ }^{5,6}$ The relationship between variability in the $\mathrm{SBP}$ and the progression of CAC has not been investigated. The aim of this study was to investigate the association between visit-to-visit variability in the SBP and the progression of CAC.

\section{METHODS}

Patients and study design

We performed a retrospective cohort study with 164 participants at the Department of Cardiology Yamashiro Public Hospital (Kyoto, Japan) from
April 2006 to March 2012. The inclusion criterion was a clinical suspicion of coronary artery disease. All the participants were in stable clinical condition and agreed to participate in the investigation.

Type 2 diabetes was diagnosed according to the Report of the Expert Committee on the Diagnosis and Classification of Diabetes Mellitus.? The body mass index (BMI) was calculated as the weight in kilograms divided by the height in meters squared. The patients were classified as nonsmokers, past smokers or current smokers according to a self-administered questionnaire. The patients with advanced renal dysfunction (serum $\mathrm{Cr}$ over $2.0 \mathrm{mg} \mathrm{dl}^{-1}$ ) or malignancy, those with major cardiovascular event during the follow-up and those with any changes in medication for hypertension during the year of data collection were excluded from this study. A cardiovascular event was defined as the presence of a myocardial infarction, cerebral infarction or angina that required a revascularization.

We then evaluated the relationship between variability in the SBP and the progression of CAC. This study was approved by the local Research Ethics Committee and conducted in accordance with the Declaration of Helsinki, and informed consent was obtained from all of the participants.

\section{Measurement of the blood pressure and data collection}

We measured the blood pressure at every visit for a year and calculated the visit-to-visit variability in blood pressure, as described below. After collecting the blood pressure data, we performed MSCT, and blood samples were

${ }^{1}$ Department of Endocrinology and Metabolism, Kyoto Prefectural University of Medicine, Graduate School of Medical Science, Kyoto, Japan; ${ }^{2}$ Department of Cardiology, Yamashiro Public Hospital, Kyoto, Japan and ${ }^{3}$ Department of Endocrinology and Metabolism, Yamashiro Public Hospital, Kyoto, Japan

Correspondence: Dr M Fukui, Department of Endocrinology and Metabolism, Kyoto Prefectural University of Medicine, Graduate School of Medical Science, 465 Kajii-cho, Kawaramachi-Hirokoji, Kamigyo-ku, Kyoto 602 8566, Japan.

E-mail: sayarinapm@hotmail.com

Received 19 October 2012; revised 29 March 2013; accepted 15 April 2013; published online 4 July 2013 
collected as baseline data. In a follow-up study, we performed MSCT again and calculated the progression of the CAC score.

The sitting blood pressure was measured after a 5-min rest in a quiet space at every visit over the course of a year using a device (HEM-906: OMRON, Kyoto, Japan) that is able to automatically measure blood pressure. The visitto-visit variability in SBP was calculated as follows: coefficient of variation $(C V)=$ s.d. of SBP $\times 100$ /average SBP. We calculated the CV and s.d. of the SBP, as well as the diastolic blood pressure (DBP) or pulse pressure, as parameters of the blood pressure variability.

Blood samples were taken in the morning at baseline. The serum total cholesterol, high-density lipoprotein (HDL) cholesterol and triglyceride concentrations were assessed using standard enzymatic methods. The serum low-density lipoprotein (LDL) cholesterol concentration was calculated using the Friedewald formula (LDL cholesterol=total cholesterol - HDL cholesterol - triglycerides/5). ${ }^{8}$

The progression of the CAC score was calculated as follows: progression of CAC score $=$ increase of CAC score/follow-up duration (year).

\section{Measurement of the CAC score}

We performed MSCT (Aquillion 64, Toshiba Medical, Tokyo, Japan) twice at baseline and at the end of a follow-up interval. The calcification scoring parameters included the $2-\mathrm{mm}$ collimation width, a gantry rotation speed of $0.4 \mathrm{~s}$ per rotation, tube energy of $120 \mathrm{kV}$, and an effective tube current of $400 \mathrm{~mA}$, using prospective ECG-gated axial scanning. The total CAC scores were determined on a workstation (ZIO Station, ZIO Soft, Tokyo, Japan) with software to determine the CAC using the Agatston method, as previously described. ${ }^{9}$ Two experienced observers who were blinded to the identity and clinical presentation of the participant analyzed the CAC scores.

\section{Statistical analysis}

The mean values and frequencies of the potential confounding variables were calculated. Because triglycerides and the progression of the CAC score showed skewed distributions, log transformations were carried out before performing the correlation and regression analysis. The progression of the CAC score was log-transformed after adding 1 . The relationship between the visit-to-visit variability in SBP and the progression of the CAC score, as well as the relationship between the visit-to-visit variability in SBP and age or other variables, were examined using Pearson's correlation analysis. To examine the effects of various factors on the logarithm of the progression of the CAC score +1 , the following factors were considered simultaneously as independent variables for the multiple regression analyses: age, sex, follow-up duration, BMI, average SBP, heart rate, hemoglobin Alc, total cholesterol, logarithm of triglycerides, uric acid, smoking status, antihypertensive drug, statin and visitto-visit variability, as well as the CV and s.d. of the SBP, DBP or pulse pressure. All of the continuous variables are presented as the mean \pm s.d. or as the absolute number. A $P$-value $<0.05$ was considered as significant.

\section{RESULTS}

The characteristics of the 164 patients enrolled in this study are shown in Table 1. The mean CV of the SBP was $6.12 \pm 4.16 \%$. The average number of blood pressure measurements per subject during the yearlong data collection period was $7.02 \pm 2.40$. The average follow-up duration was $3.93 \pm 1.36$ years. The relationship between the CV of the SBP and the other variables are shown in Table 2. No significant correlations were found between the $\mathrm{CV}$ of the SBP and the average SBP, heart rate, hemoglobin A1c, total cholesterol, LDL cholesterol and uric acid. The CV of the SBP correlated positively with the logarithm of the progression of the CAC score $+1 \quad(r=0.4382$, $P<0.0001)$. A simple correlation and multiple regression analysis on the logarithm of the progression of the CAC score +1 is shown in Table 3. Multiple regression analysis demonstrated that the CV of the SBP $(\beta=0.3826, P<0.0001)$ was independently associated with the logarithm of the progression of the CAC score +1 . Moreover, the s.d. of the SBP $(\beta=0.3894, P<0.0001)$, the $\mathrm{CV}$ of the DBP $(\beta=0.2218$,

\section{Table 1 Characteristics of patients at baseline}

n

Sex (male/female)

$81 / 83$

Duration of follow up (year)

$3.93(1.36)$

Body mass index $\left(\mathrm{kg} \mathrm{m}^{-2}\right)$

$23.6(4.3)$

Average arms SBP $(\mathrm{mm} \mathrm{Hg})$

$132.2(16.2)$

Average arms DBP $(\mathrm{mm} \mathrm{Hg})$

$74.2(9.4)$

Average pulse pressure $(\mathrm{mm} \mathrm{Hg})$

$58.2(12.2)$

Coeffeicient of variation of SBP (\%)

$6.12(4.16)$

$8.10(4.67)$

Coeffeicient of variation of pulse pressure (\%)

$12.1(7.82)$

Heart rate

$75.6(11.1)$

$5.92(1.51)$

Hemoglobin Alc (\%)

$5.03(0.96)$

$2.96(0.83)$

$1.37(0.36)$

$1.62(0.92)$

331.1 (86.8)

$72.5(41.5)$

$139 / 25$

$112 / 32 / 20$

$62 / 20 / 13 / 50 /$

Statin $( \pm)$

39.8 (99.3)

Abbreviations: ACE, angiotensin-converting enzyme; ARB, angiotensin II receptor blocker; CAC, coronary artery calcification; DBP, diastolic blood pressure; SBP, systolic blood pressure. Data are expressed as mean (s.d.) or absolute number.

Table 2 Correlation between coefficient of variation of systolic blood pressure and other variables

\begin{tabular}{lrr}
\hline & r-value & P-value \\
\hline Age & 0.2499 & 0.0012 \\
Duration of follow-up & 0.2212 & 0.0042 \\
Body mass index & -0.1815 & 0.0193 \\
Average systolic blood pressure & 0.0329 & 0.6740 \\
Heart rate & 0.0412 & 0.5981 \\
Hemoglobin Alc & 0.0686 & 0.3799 \\
Total cholesterol & 0.1160 & 0.2623 \\
Low-density lipoprotein cholesterol & -0.1353 & 0.0821 \\
High-density lipoprotein cholesterol & -0.2018 & 0.0091 \\
Logarithm of triglycerides & 0.2139 & 0.0056 \\
Uric acid & 0.1440 & 0.0641 \\
Logarithm of progression of CAC score & 0.4382 & $<0.0001$
\end{tabular}

Abbreviation: CAC, coronary artery calcification.

$P=0.0042)$ and the s.d. of the DBP $(\beta=0.2028, P=0.0090)$ were also independently associated with the logarithm of the progression of the CAC score +1 .

The CV $(\beta=0.0657, P=0.3978)$ and s.d. $(\beta=0.0889, P=0.2631)$ of the pulse pressure were not independently associated with the logarithm of the progression of the CAC score +1 .

\section{DISCUSSION}

The major finding of our study is that the visit-to-visit variability in the SBP is a risk factor for the progression of CAC, which is a good surrogate marker of the presence and severity of coronary 
Table 3 Simple correlation and multiple regression analyses on logarithm of progression of coronary artery calcification score

\begin{tabular}{|c|c|c|c|c|}
\hline & r-value & P-value & $\beta$ & $\mathrm{P}$-value \\
\hline Age & 0.1552 & 0.0386 & 0.1261 & 0.1375 \\
\hline Sex & - & - & 0.1573 & 0.0579 \\
\hline Duration of follow-up & 0.2016 & 0.0058 & 0.0572 & 0.4169 \\
\hline Body mass index & 0.0196 & 0.7934 & 0.1033 & 0.2287 \\
\hline Average systolic blood pressure & 0.2710 & 0.0004 & 0.2387 & 0.0014 \\
\hline CV of systolic blood pressure & 0.4382 & $<0.0001$ & 0.3826 & $<0.0001$ \\
\hline Heart rate & 0.1434 & 0.0065 & 0.0877 & 0.2341 \\
\hline Hemoglobin A1c & 0.2450 & 0.0010 & 0.2000 & 0.0054 \\
\hline Total cholesterol & -0.0481 & 0.5211 & 0.1041 & 0.1756 \\
\hline Logarithm of triglycerides & 0.0067 & 0.9297 & -01534 & 0.0661 \\
\hline Uric acid & 0.1686 & 0.0240 & 0.1002 & 0.1890 \\
\hline Smoking status & - & - & -0.0154 & 0.8352 \\
\hline Calcium-channel blocker & - & - & -0.0070 & 0.9213 \\
\hline Diuretic drug & - & - & 0.0080 & 0.2685 \\
\hline ACE inhibitor & - & - & -0.1364 & 0.0639 \\
\hline ARB & - & - & -0.0624 & 0.4160 \\
\hline alpha blocker & - & - & 0.0363 & 0.6394 \\
\hline beta blocker & - & - & -0.0437 & 0.5294 \\
\hline Statin & - & - & 0.1472 & 0.0559 \\
\hline
\end{tabular}

Abbreviations: ACE, angiotensin-converting enzyme; ARB, angiotensin II receptor blocker; CV, coefficient of variation. Sex was defined as female $(=0)$ or male $(=1)$, smoking status was defined as nonsmoker $(=0)$, past smoker $(=1)$, or current smoker $(=2)$, and medication for hypertension or dyslipidemia was defined as without $(=0)$ or with $(=1)$.

atherosclerosis. Although previous studies have reported that the average SBP is an independent risk factor for atherosclerosis, recent studies have suggested that both the average SBP and the variability in SBP are independent risk factors for atherosclerosis and organ damage in patients with hypertension and with treated hypertension. ${ }^{3,4,10,11}$ Moreover, Rothwell et al. ${ }^{3}$ suggested that the average SBP was a weak predictor of stroke and coronary events, whereas the visit-to-visit variability in SBP was a strong predictor of both.

Some studies have reported that the CAC score is a better predictor of subsequent CVD events. The CAC score by MSCT can be related to the extent and severity of coronary atherosclerotic disease and can improve the prediction of the risk of CHD (coronary heart disease). ${ }^{12,13}$ In our study, multiple regression analysis revealed that the CV of the SBP was more predictive than was the average SBP for the progression of CAC.

Because variability in the SBP might be influenced by other risk factors associated with the calcium score, we have assessed the relationship between the CV of the SBP and other risk factors. Age, follow-up duration, BMI, HDL cholesterol and the logarithm of triglycerides were correlated with the CV of the SBP. Moreover, variability in the SBP may be affected by antihypertensive drugs, including angiotensin-converting enzyme inhibitors, angiotensin II receptor blockers and beta blockers. ${ }^{14}$ However, variability in the SBP was independently associated with the progression of the CAC score, even after making adjustments for age, follow-up duration, BMI, the logarithm of triglycerides and antihypertensive drugs in this study.

Variability in the SBP is a dynamic and complex phenomenon that arises from intricate interactions among behavioral, environmental, humoral, and neural central or reflex pathways. ${ }^{15}$ For example, postural changes, prandial effects and psychological stress, as well as the effects of humoral factors, such as angiotensin II, bradykinin or nitric oxide, increased the central sympathetic drive and reduced the arterial compliance. ${ }^{15}$ On the other hand, it was reported that the visit-to-visit variability was the result of improper titration of the antihypertensive therapy and improper downward titration of the antihypertensive drug regimen over the summer. ${ }^{16}$ Consequently, the patients in our study who underwent any changes in their medication for hypertension during the data collection period were excluded.

The structural vessel abnormalities resulted mainly from a biochemical derangement, and one possible mechanism connecting the blood pressure variability with structural vessel abnormalities is that an increased blood pressure variability might be related to a greater traumatic effect of wider blood pressure swings on the vessel wall, promoting target-organ damage. ${ }^{17}$ Eto et al. ${ }^{18}$ suggested that increased blood pressure variability, independent of the average blood pressure level, impairs endothelial function by inhibiting nitric oxide production and enhances neointimal formation, and may thereby contributes to atherogenesis in animal models. A greater blood pressure variability has also been attributed to increased arterial stiffness. Kikuya et al. ${ }^{19}$ suggested that increased arterial stiffness might cause an increase in the blood pressure variability.

There were several parameters of the variability in blood pressure other than the CV of the SBP. To assess whether these other parameters of the variability in blood pressure could be linked with the progression of the CAC score, we have incorporated the s.d. of the SBP, the CV or s.d. of the DBP and the CV or s.d. of the pulse pressure into multiple regression analyses. The multiple regression analysis demonstrated that the s.d. of the SBP, the CV of the DBP and the s.d. of the DBP were also independently associated with the progression of the CAC score. However, the CV and s.d. of the pulse pressure were not independently associated with the progression of the CAC score.

There are some limitations in our study. First, the study population consisted of Japanese men and women; therefore, it is uncertain whether these findings can be generalized to other ethnic groups. Second, medication non-adherence might have been one of the causes of visit-to-visit blood pressure variation; however, we have no data on medication adherence. Finally, we have no data on the reproducibility of the MSCT data. Moreover, a previous study has reported that the reproducibility of MSCT remains insufficient. ${ }^{20}$

Previous studies have emphasized hypertension, diabetes, smoking and dyslipidemia as risk factors for the progression of atherosclerosis. We suggest that it is important to consider the variability in blood pressure and clarify its role in preventing the progression of coronary atherosclerosis. To the best of our knowledge, this is the first study to investigate the relationship between visit-to-visit variability in the SBP and the progression of CAC. Large prospective trials are needed to better assess this relationship.

\section{CONCLUSIONS}

In conclusion, visit-to-visit variability in the SBP could be a novel risk factor for the progression of CAC.

\section{CONFLICT OF INTEREST}

The authors declare no conflict of interest.

1 Isomaa B, Almgren P, Tuomi T, Forsén B, Lahti K, Nissén M, Taskinen MR, Groop L. Cardiovascular morbidity and mortality associated with the metabolic syndrome. Diabetes Care 2001; 24: 683-689.

2 Multiple Risk Factor Intervention Trial Research Group. Multiple risk factor intervention trial. Risk factor changes and mortality results. JAMA 1982; 248: 1465-1477.

3 Rothwell PM, Howard SC, Dolan E, O'Brien E, Dobson JE, Dahlöf B, Sever PS, Poulter NR. Prognostic significance of visit-to-visit variability, maximum systolic blood pressure, and episodic hypertension. Lancet 2010; 375: 895-905. 
4 Okada H, Fukui M, Tanaka M, Inada S, Mineoka Y, Nakanishi N, Senmaru T, Sakabe K, Ushigome E, Asano M, Yamazaki M, Hasegawa G, Nakamura N. Visit-to-visit variability in systolic blood pressure is correlated with diabetic nephropathy and atherosclerosis in patients with type 2 diabetes. Atherosclerosis 2012; 220: 155-159.

5 Baumgart D, Schmermund A, Goerge G, Haude M, Ge J, Adamzik M, Sehnert C, Altmaier K, Groenemeyer D, Seibel R, Erbel R. Comparison of electron beam computed tomography with intracoronary ultrasound and coronary angiography for detection of coronary atherosclerosis. J Am Coll Cardiol 1997; 30: 57-64.

6 Nallamothu BK, Saint S, Bielak LF, Sonnad SS, Peyser PA, Rubenfire M, Fendrick AM. Electron-beam computed tomography in the diagnosis of coronary artery disease: a meta-analysis. Arch Intern Med 2001; 161: 833-838.

7 Expert Committee on the Diagnosis and Classification of Diabetes Mellitus. Report of the expert committee on the diagnosis and classification of diabetes mellitus. Diabetes Care 2003; 26: S5-20.

8 Friedewald WT, Levy RI, Fredrickson DS. Estimation of the concentration of low-density lipoprotein cholesterol in plasma, without use of the preparative ultracentrifuge. Clin Chem 1972; 18: 499-502.

9 Agatston AS, Janowitz WR, Hildner FJ, Zusmer NR, Viamonte M Jr, Detrano R. Quantification of coronary artery calcium using ultrafast computed tomography. J Am Coll Cardiol 1990; 15: 827-832.

10 Tatasciore A, Renda G, Zimarino M, Soccio M, Bilo G, Parati G, Schillaci G, De Caterina R. Awake systolic blood pressure variability correlates with target-organ damage in hypertensive subjects. Hypertension 2007; 50: 325-332.

11 Miao CY, Xie HH, Zhan LS, Su DF. Blood pressure variability is more important than blood pressure level in determination of end-organ damage in rats. $J$ Hypertens 2006; 24: 1125-1135.

12 Rumberger JA, Brundage BH, Rader DJ, Kondos G. Electron beam computed tomographic coronary calcium scanning: a review and guidelines for use in asymptomatic persons. Mayo Clin Proc 1999; 74: 243-252.

13 Budoff MJ, Achenbach S, Blumenthal RS, Carr JJ, Goldin JG, Greenland P, Guerci AD, Lima JA, Rader DJ, Rubin GD, Shaw LJ, Wiegers SE. American Heart
Association Committee on Cardiovascular Imaging and Intervention; American Heart Association Council on Cardiovascular Radiology and Intervention; American Heart Association Committee on Cardiac Imaging, Council on Clinical Cardiology. Assessment of coronary artery disease by cardiac computed tomography: a scientific statement from the American Heart Association Committee on Cardiovascular Imaging and Intervention, Council on Cardiovascular Radiology and Intervention, and Committee on Cardiac Imaging, Council on Clinical Cardiology. Circulation 2006; 114 1761-1791.

14 Webb AJ, Fischer U, Mehta Z, Rothwell PM. Effects of antihypertensive-drug class on interindividual variation in blood pressure and risk of stroke: a systematic review and meta-analysis. Lancet 2010; 375: 906-915.

15 Parati G, Ochoa JE, Bilo G. Blood pressure variability, cardiovascular risk, and risk for renal disease progression. Curr Hypertens Rep 2012; 14: 421-431.

16 Modesti PA, Morabito M, Bertolozzi I, Massetti L, Panci G, Lumachi C, Giglio A, Bilo G, Caldara G, Lonati L, Orlandini S, Maracchi G, Mancia G, Gensini GF, Parati G. Weatherrelated changes in 24-hour blood pressure profile: effects of age and implications for hypertension management. Hypertension 2006; 47: 155-161.

17 Warlow C, Sudlow C, Dennis M, Wardlaw J, Sandercock P. Stroke. Lancet 2003; 362 : $1211-1224$.

18 Eto M, Toba K, Akishita M, Kozaki K, Watanabe T, Kim S, Hashimoto M, Sudoh N, Yoshizumi M, Ouchi Y. Reduced endothelial vasomotor function and enhanced neointimal formation after vascular injury in a rat model of blood pressure lability. Hypertens Res 2003; 26: 991-998.

19 Kikuya M, Hozawa A, Ohokubo T, Tsuji I, Michimata M, Matsubara M, Ota M, Nagai K, Araki T, Satoh H, Ito S, Hisamichi S, Imai Y. Prognostic significance of blood pressure and heart rate variabilities: the Ohasama study. Hypertension 2000; 36: 901-906.

20 Leber AW, Becker A, Knez A, von Ziegler F, Sirol M, Nikolaou K, Ohnesorge B, Fayad ZA, Becker CR, Reiser M, Steinbeck G, Boekstegers P. Accuracy of 64-slice computed tomography to classify and quantify plaque volumes in the proximal coronary system: a comparative study using intravascular ultrasound. J Am Coll Cardiol 2006; 47: 672-677. 